\title{
The cloud of gas falling toward the central black hole in the milky way
}

\author{
J. Miralda-Escudé $1,2, \mathrm{a}$ \\ ${ }^{1}$ Institució Catalana de Recerca i Estudis Avançats, Barcelona, Catalonia \\ ${ }^{2}$ Institut de Ciències del Cosmos, Universitat de Barcelona, Catalonia
}

\begin{abstract}
The cloud of gas that will pass within $200 \mathrm{AU}$ of the central black hole of our Galaxy in 2013 may be generated by a disk around an old, low-mass star that was created in a tidal encounter with one of the stellar black holes that are expected to accumulate in the central region of the stellar cusp.
\end{abstract}

\section{WHY THIS CLOUD IS SO INTERESTING}

A strange cloud was reported by $[1,2]$ that is falling toward the central black hole on a highly eccentric orbit. The cloud will reach peribothron in September of 2013 at a distance of only $180 \mathrm{AU}$, and its apobothron is at about $10000 \mathrm{AU}$, with an orbital period of 200 years. From the observed infrared continuum and recombination line emission and its resolved angular size, the cloud is inferred to have a mass of a few Earth masses and a size of $100 \mathrm{AU}$, which has been recently stretched as the cloud is disrupted by the gravitational tide of the central black hole.

The cloud is interesting because the evolution of the debris over the next few years will become a probe to the structure of the hot medium around the central black hole. In addition, the origin of the cloud is a mystery being debated at present, and may be related to important clues on the physical processes in the Galactic center. Here, I will summarize the model that I have recently proposed for how this cloud was made ([3]), and describe the reasons that in my opinion make this model a plausible one.

\section{THE MODEL OF A STAR-STELLAR BLACK HOLE ENCOUNTER}

This model proposes that the cloud contains in its interior an old, low-mass main-sequence star with a gas disk around it. Every time the star with its disk passes through the peribothron of its orbit around the central black hole, tidal shocks induced in the outer edge of the disk cause the ejection of gas which creates the cloud that we observe. The disk is limited to a size of about 1 AU from this tidal perturbation at each peribothron. The cloud is assumed to be recreated at every orbit from the small fraction of the disk mass that is pulled out during one peribothron passage and is left orbiting the star-disk system, which is then photoionized by the ambient ionizing radiation field. Photoionization of a large disk around a young star was proposed by [4], but their model has the difficulty of explaining how a young star can be deflected into the observed eccentric orbit without destroying the disk. In my model the disk is much smaller and the photoionization wind arises from the tidal gaseous debris that are recreated at every orbit.

\footnotetext{
a e-mail: miralda@icc.ub.edu
}

How was this strange system of a disk around an old star formed? The model hypothesizes that a close encounter occurred between a typical stellar black hole (with $\sim 10 M_{\odot}$ ) and an old main-sequence star. Both the stellar black hole and the main-sequence star were moving on random orbits within the stellar cusp around the central black hole. Their close encounter occurred at some point along the present orbit of the cloud, at a small enough impact parameter to lift some matter from the star, but not so small to completely destroy the star. Some of the torn material can then fall back to the star and form a disk. The star is also strongly spun up by the encounter, and the angular momentum of the star can be slowly transferred to the disk after the encounter and allow for a large disk expansion. Since this event took place, the star has been moving on the present orbit of the cloud, with its disk gradually pushing matter out to the tidal edge of about 1 AU through the usual mechanism of angular momentum transfer as more angular momentum is absorbed by the disk from the rapidly rotating star.

Naturally, one may be left wondering if this intricate and complex series of events is really justified as a particularly likely scenario just to explain a cloud of gas. Nevertheless, the attractiveness of this model is that one can estimate the rate at which this class of encounters of stellar black holes and main-sequence stars should be ocurring, and the only place where they are expected to be relatively frequent is precisely where this cloud is observed. Stellar black holes created by the collapse of massive stars over the entire history of the bulge within 5 parsecs of the center should have migrated inward by dynamical friction, so their abundance is greatly enhanced near the center, yielding an estimated encounter rate of $10^{-6}$ per year. So, if the resulting cloud is produced on many orbits after the encounter, there can be a reasonable probability to observe one of these systems at a random time.

\section{WHAT FUTURE OBSERVATIONS CAN TELL US}

The cloud is now being disrupted and will produce extended gas filaments, which will provide a remarkable probe to the distribution of the hot medium around the central black hole (see the simulations of [5]). The evolution of the extended debris, however, will not test the difference between this model and the possibility of an isolated cloud, because if a central star is present, the 
cloud of gas is by now already unbound and being tidally disrupted. The distinctive prediction that we expect with a central star is that an unresolved source of emission should at some point be observed exactly along the Keplerian orbit measured for the cloud before the peribothron passage. In addition, the tidal shocks on the disk during peribothron might produce an infrared flare which would be a unique feature of this model.

If the observations were to support this model, this would open an interesting window into the stellar population and the type of collisions that take place in the Galactic nucleus.

\section{References}

[1] Gillessen, S., Genzel, R., Fritz, T. K., et al. 2012, Nature, 481, 51

[2] Gillessen, S., Genzel, R., Fritz, T. K., et al. 2012, submitted to ApJ (arXiv:1209.2272).

[3] Miralda-Escudé, J. 2012, ApJ, 756, 86.

[4] Murray-Clay, R. A., \& Loeb, A. 2012, NatCo, 3, 1049 (arXiv:1112.4822)

[5] Schartmann, M., Burkert, A., Alig, C., Gillessen, S., Genzel, R., Eisenhauer, F., \& Fritz, T. K. 2012, ApJ, 755, 155 . 